\section{YOUSSEF} ABDEL MASSIH

CNRS libanais

Centre de recherches géophysiques Bhannes, Liban dalia@cnrs.edu.lb

\section{A.-H. SOUBRA}

Institut de recherche en génie civil et mécanique Université de Nantes Bd de I'Université, BP 152 44603 Saint-Nazaire Cedex Abed.soubra@univ-nantes.fr

\title{
Effet de la variabilité spatiale du sol dans l'étude du comportement des fondations superficielles filantes
}

Cet article présente une analyse probabiliste à l'état limite ultime d'une fondation superficielle filante soumise à une charge verticale centrée et posée sur un sol présentant une variabilité spatiale. La cohésion et l'angle de frottement interne sont modélisés à l'aide de champs aléatoires non gaussiens et anisotropes en ce qui concerne la distance d'autocorrélation. La méthode de discrétisation de champ employée est la méthode de représentation spectrale. Les modèles déterministes sont basés sur des simulations numériques utilisant le logiciel FLAC ${ }^{3 D}$. Des échantillons de sol ont été générés et la charge ultime (i.e. réponse du système) correspondant à chaque réalisation de sol a été calculée. La moyenne et l'écart-type de la réponse de toutes les simulations ont été déterminés. Il a été montré que la variabilité spatiale du sol crée une asymétrie dans le champ de vitesses et de déformations et aboutit à une rotation de la fondation pour une réalisation donnée du champ aléatoire. Cependant, la rotation moyenne de la fondation, issue de I'ensemble des simulations de Monte-Carlo, est nulle. La loi lognormale est celle qui ajuste au mieux l'histogramme de la charge ultime de la fondation, obtenu à partir des simulations de Monte-Carlo. La valeur de la moyenne de la charge ultime de toutes les simulations est inférieure à la valeur déterministe calculée pour un sol homogène dont les caractéristiques de cisaillement sont constantes et égales aux valeurs moyennes. La moyenne de la charge ultime diminue, puis augmente avec l'augmentation des distances d'autocorrélation pour un sol isotrope. Un minimum existe pour une distance d'autocorrélation égale à la largeur de la fondation. La charge ultime est plus sensible aux variations de l'angle de frottement interne qu'à celles de la cohésion.

Mots-clés : fondations superficielles, charge ultime, champs aléatoires, représentation spectrale, simulations de Monte-Carlo. 


\section{Effect of soil spatial variability on the behavior of shallow strip footings}

The paper presents the effect of the soil spatial variability on the behavior of a vertically loaded strip footing at the ultimate limit state. The soil cohesion and angle of internal friction are modelled as non-gaussian anisotropic random fields concerning the autocorrelation distance. The spectral representation method is used for the discretization of the random fields. The deterministic models employed in the paper are based on numerical simulations using FLAC ${ }^{3 D}$ software. Several soil samples were generated and the ultimate footing load (i.e. system response) corresponding to each sample was computed. The mean and standard deviation of the system response were determined. It was shown that the soil spatial variability makes the velocity field unsymmetrical for a given realisation of the random field and it leads to a rotation of the foundation. However, the mean value of the footing rotation obtained from all the Monte-Carlo simulations is null. The lognormal distribution is the one that better fits the histogram of the ultimate footing load. The average footing load of a spatially random soil, computed based on the different samples of the random field, is lower than the deterministic value obtained for a homogeneous soil using the mean values of the random parameters. A critical case appears when the autocorrelation distance is equal to the footing breadth, the mean ultimate footing load being minimal in that case. The ultimate footing load was found more sensitive to the angle of internal friction of the soil than to the soil cohesion.

Key words: shallow foundations, ultimate load, random fields, spectral representation, Monte-Carlo simulations.

\section{Introduction}

La variabilité naturelle des propriétés du sol affecte le comportement des ouvrages en géotechnique (portance et tassement des fondations, stabilité des pentes, etc.). La prise en compte de cette variabilité spatiale dans les modèles mécaniques requiert une modélisation de ces propriétés par des champs aléatoires. Leur discrétisation permet d'effectuer un couplage mécanofiabiliste pour le système étudié.

Plusieurs auteurs ont considéré l'effet de la variabilité spatiale du sol dans leurs modèles de calcul. On peut citer (i) Griffiths et Fenton (1993) dans les problèmes d'écoulement, (ii) Fenton et Griffiths (2002, 2005) dans les problèmes de tassement, (iii) Assimaki et al. (2002) dans les problèmes de propagation des ondes sismiques, (iv) Griffiths et Fenton (2000) dans les problèmes de stabilité de pentes et (v) Fenton et Griffiths (2003), Griffiths et Fenton (2001), Griffiths et al. (2002), Kalfa (2006), Popescu et al. (2005), Soubra et Youssef Abdel Massih (2008), Soubra et al. (2008) et Youssef Abdel Massih (2007) dans les problèmes de portance de fondations. D'autres auteurs ont modélisé la variabilité des propriétés du sol par des variables aléatoires caractérisées par des lois de distribution de probabilité. Dans ces approches, un paramètre aléatoire donné a une valeur constante en tout point du sol et la variabilité du sol est prise en compte d'une simulation à une autre en supposant que le sol est homogène pour chaque simulation. Ces méthodes ne considèrent pas la variabilité spatiale des propriétés du sol. On peut citer, dans le cadre de ces approches, les récents travaux de Soubra (2009), Soubra et Youssef Abdel Massih (2007), Youssef Abdel Massih et Soubra $(2007,2008)$ et Youssef Abdel Massih et al. (2008a, b).

Cet article présente une analyse probabiliste à l'état limite ultime d'une fondation superficielle filante soumise à un chargement vertical centré et posée sur un sol présentant une variabilité spatiale. La cohésion et l'angle de frottement interne sont modélisés à l'aide de champs aléatoires non gaussiens et anisotropes en ce qui concerne la distance d'autocorrélation. La méthode de discrétisation de champs employée est la méthode de représentation spectrale. Les simulations numériques déterministes sont basées sur un modèle élastoplastique utilisant le logiciel FLAC ${ }^{3 D}$. On présente successivement la méthodologie employée pour la discrétisation des champs aléatoires et la représentation des réalisations de champs ainsi qu'une comparaison entre les fonctions statistiques cibles des champs et 
celles générées, le modèle probabiliste de calcul de la réponse du système (i.e. charge ultime de la fondation), et enfin les résultats probabilistes obtenus.

\section{2}

\section{Méthode de discrétisation et réalisations d'un champ aléatoire}

La méthode de représentation spectrale décrite par Shinozuka et Deodatis (1991) et Popescu et al. (1998) a été utilisée pour la génération de champs aléatoires respectant une fonction de densité spectrale et une densité de probabilité prescrites. Cette méthode permet la discrétisation de champs non gaussiens et anisotropes à l'aide de transformations de Fourrier et d'une transformation non linéaire couplée avec un processus itératif. On a ainsi réalisé la génération d'un champ aléatoire à deux variables indépendantes représentant la cohésion $c$ et l'angle de frottement interne $\varphi$ du sol. Une génération d'un échantillon $(c, \varphi)$ représente une configuration de sol où la valeur d'un paramètre dépend de sa position. Le sol ainsi généré reproduit les caractéristiques statistiques caractérisant le sol réel. Évidemment, l'analyse d'un seul échantillon ne suffit pas à tirer des conclusions sur le sol réel puisque le sol généré n'est qu'une réalisation probable reproduisant les statistiques du sol réel. La génération de champ aléatoire acquiert tout son intérêt dans sa mise en œuvre au cours de simulations par la méthode de Monte-Carlo. Un nombre significatif d'échantillons de sol doit être généré afin d'avancer des conclusions. Par exemple, pour l'étude de la capacité portante d'une fondation, cela revient à générer un grand nombre d'échantillons de sol, chacun ayant des paramètres c et $\varphi$ qui ne sont pas constants et dépendent de la position dans le sol, puis à déterminer pour chaque échantillon avec un logiciel comme FLAC ${ }^{3 \mathrm{D}}$, la capacité portante. Ce n'est alors que la moyenne issue de l'ensemble des valeurs de capacités portantes calculées pour tous les échantillons de sol qui est interprétable.

En 2D, la formule de discrétisation d'un champ gaussien $\mathrm{mV}$-nD (i.e. à $\mathrm{m}$ variables et à $\mathrm{n}$ dimensions) s'écrit dans le domaine spatial et dans le cas d'une seule variable à deux dimensions comme suit (Yamazaki et Shinozuka, 1988):

$$
\begin{gathered}
f_{G r}\left(x_{p}, y_{q}\right)=\operatorname{Re}\left[\sqrt{2} \sum_{k=0}^{M_{x}-1}\left(\sum_{i=0}^{M_{y}-1} A_{k j} e^{i \phi_{k}} e^{i \kappa_{y} y_{q}}\right) e^{i K_{x} x_{p}}\right] \\
+\operatorname{Re}\left[\sqrt{2} \sum_{k=0}^{M_{x}-1}\left(\sum_{t=0}^{M_{y}-1} A_{k i} e^{i \Psi_{k}} e^{-i K_{y} y_{q}}\right) e^{i K_{x} x_{p}}\right]
\end{gathered}
$$

où Re est la partie réelle d'un nombre complexe, $x_{p}=p \Delta x$ avec $\left(p=0,1, \ldots, M_{x}-1\right)$ et $y_{q}=q \Delta y$ avec $\left(q=0,1, \ldots, M_{y}-1\right)$.

$M_{x}$ et $M_{y}$ sont respectivement les nombres de points simulés suivant $\mathrm{x}$ et $\mathrm{y}$ dans le domaine spatial, $(\Delta \mathrm{x}, \Delta \mathrm{y})$ sont les dimensions du maillage dans le domaine spatial, $\phi_{k l}$ et $\psi_{k l}$ sont des phases aléatoires indépendantes uniformément reparties entre 0 et $2 \pi$.

Dans l'équation (1), le terme $A_{\mathrm{kl}}$ est donné par :

$$
A_{k l}=\sqrt{2 S_{f f}\left(\kappa_{x, k}, \kappa_{y, l}\right) \Delta \kappa_{x} \Delta \kappa_{y}}
$$

où $\left(\kappa_{\mathrm{x}, \mathrm{k}^{\prime}} \kappa_{\mathrm{y}, \mathrm{l}}\right)$ sont les coordonnées des points simulés dans le domaine fréquentiel, $\left(\Delta \kappa_{x}, \Delta \kappa_{y}\right)$ représentent les dimensions du maillage dans le domaine fréquentiel ; $\mathrm{S}_{\mathrm{ff}}\left(\kappa_{\mathrm{x}^{\prime}} \kappa_{\mathrm{y}}\right)$ est la matrice de densité spectrale évaluée en tout point du domaine fréquentiel discrétisé.

La fonction $f_{G r}$ est calculée en chaque point du domaine spatial en utilisant l'algorithme de la transformation de Fourrier rapide (FFT) présent dans Matlab 7.0. Le champ aléatoire est ainsi estimé en des points $(\mathrm{x}, \mathrm{y})$ de l'espace. Les distances $(\Delta \mathrm{x}, \Delta \mathrm{y})$ qui représentent les dimensions du maillage spatial dépendent de la fréquence de coupure $\kappa_{u}=\left(\kappa_{x u^{\prime}} \kappa_{y u}\right)$ et du rapport entre $N$ et $M$ qui sont les nombres des points simulés dans le domaine fréquentiel et spatial respectivement. Ces distances s'expriment comme suit :

$$
\begin{aligned}
& \Delta x=\frac{2 \pi}{\kappa_{x u}} \cdot \frac{N_{x}}{M_{x}} \\
& \Delta y=\frac{2 \pi}{\kappa_{y u}} \cdot \frac{N_{y}}{M_{y}}
\end{aligned}
$$

Le rapport $\frac{\mathrm{M}}{\mathrm{N}}$ doit être au moins égal à 2 (Shinozuka et Deodatis, 1991). La valeur de $\mathrm{N}$ doit être suffisamment grande pour aboutir à une discrétisation fiable dans le domaine fréquentiel. Les valeurs suivantes : $N_{x}=N_{y}=64$ et $M_{x}=M_{y}=128$ ont été choisies dans cette étude (Shinozuka et Deodatis, 1991).

Il est à noter qu'un champ aléatoire à deux variables indépendantes peut être représenté par deux champs aléatoires indépendants chacun à une variable. Ainsi, les deux fonctions $\mathrm{f}_{G \mathrm{r}}$ décrivant la discrétisation des deux champs sont générées indépendamment l'une de l'autre.

\section{6.}

\section{Réalisations des champs des propriétés du sol}

Une fonction d'autocorrelation exponentielle anisotrope du premier ordre a été utilisée pour les deux paramètres aléatoires. Elle est donnée par la formule suivante (Vanmarcke, 1983) :

$$
\rho(x, y)=e^{-2 \sqrt{\left(\frac{\delta x}{\delta_{x}}\right)^{2}+\left(\frac{\delta y}{\delta_{y}}\right)^{2}}}
$$

où $\left(\delta_{x}, \delta_{y}\right)$ sont respectivement les distances d'autocorrélation horizontale et verticale et $(\delta x, \delta y)$ sont les distances horizontale et verticale qui séparent deux points quelconques du sol. La fonction densité spectrale correspondante est exprimée par la relation :

$$
S\left(\kappa_{x}, \kappa_{y}\right)=\frac{\delta_{x} \delta_{y}}{8 \pi}\left[1+\left(\frac{\delta_{x} \kappa_{x}}{2}\right)^{2}+\left(\frac{\delta_{y} \kappa_{y}}{2}\right)^{2}\right]^{-3 / 2}
$$

La fréquence de coupure $\left(\kappa_{x_{u}{ }^{\prime}} \kappa_{y_{u}}\right)$ est choisie en limitant la fonction densité spectrale à une valeur donnée en dessous de laquelle la valeur de la fonction est considérée négligeable (i.e. $\mathrm{S}\left(\mathrm{\kappa}_{\mathrm{x}_{\mathrm{u}}}, \kappa_{\mathrm{y}_{\mathrm{u}}}\right)<\varepsilon$ avec $\varepsilon=10^{-3}$ à $10^{-5}$ ). On a choisi de considérer que $\kappa_{x_{u}}=\kappa_{y_{u i}}$. Dans les paragraphes suivants, on présente une comparaison entre les fonctions de répartition et les fonctions d'autocorrélation cibles et celles générées. 


\section{(2.0)}

\section{Réalisations du champ de la cohésion}

La figure 1 présente une réalisation d'un champ isotrope lognormal de la cohésion de distance d'autocorrélation égale à $2 \mathrm{~m}\left(\delta_{x}=\delta_{y}=2 \mathrm{~m}\right)$. La moyenne et le coefficient de variation de la cohésion adoptés dans le calcul sont $20 \mathrm{kPa}$ et $20 \%$ respectivement. Les régions foncées correspondent aux petites valeurs de la cohésion et celles plus claires aux valeurs plus grandes. Les valeurs varient entre 9 et $42 \mathrm{kPa}$. La figure 2 montre la comparaison entre la fonction de probabilité cumulative (CDF) cible et celle générée du champ de la cohésion. On remarque une bonne concordance entre les deux fonctions CDF.

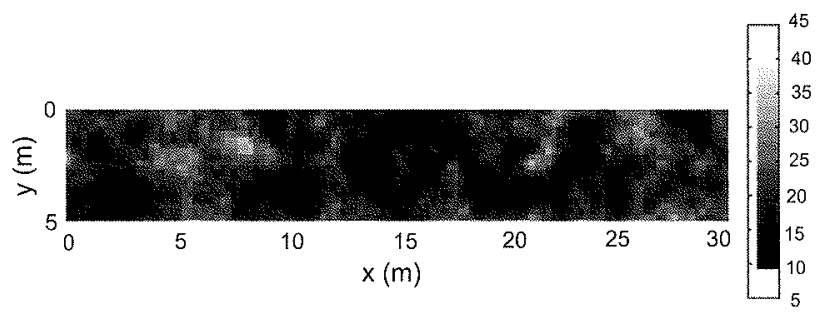

H6. 1 Réalisation du champ de la cohésion (kPa). Sample of the cohesion random field $(\mathrm{kPa})$.

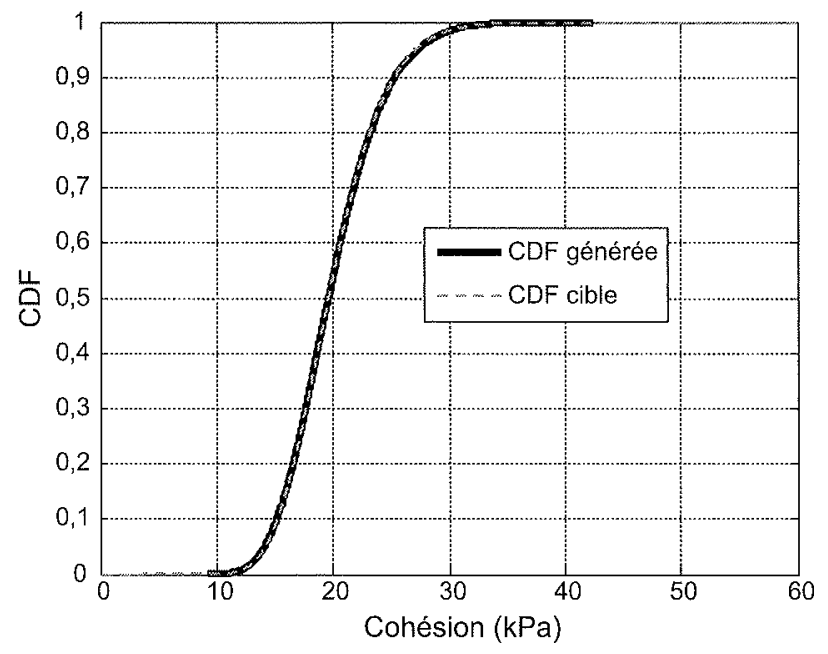

Fig. 2 Comparaison entre la fonction de répartition (CDF) cible et celle générée du champ de la cohésion.

Comparison between the target and the generated cumulative distribution function (CDF) of the cohesion random field.

La figure 3 montre une comparaison entre la fonction d'autocorrelation cible suivant une direction $\mathrm{u}$ donnée $\rho(u)=e^{-2 \sqrt{\left(\frac{\delta u}{\delta_{u}}\right)^{2}}}$ (dans laquelle ou $\mathrm{u}=\mathrm{x}$ ou $\mathrm{u}=\mathrm{y}$; ici c'est la même fonction suivant $x$ et y puisque le champ est isotrope) et celle générée, dans les deux directions $x$ et $y$, pour une réalisation donnée du champ de la cohésion. La fonction d'autocorrélation du champ généré est déterminée par la formule suivante (Fenton, 1999) :

$44 \quad \rho\left(u_{j}\right)=\frac{1}{\widehat{\sigma}^{2}(M-j)} \sum_{k=1}^{M-j}\left(X_{k}-\widehat{\mu}_{x}\right)\left(X_{k+j}-\widehat{\mu}_{x}\right)$

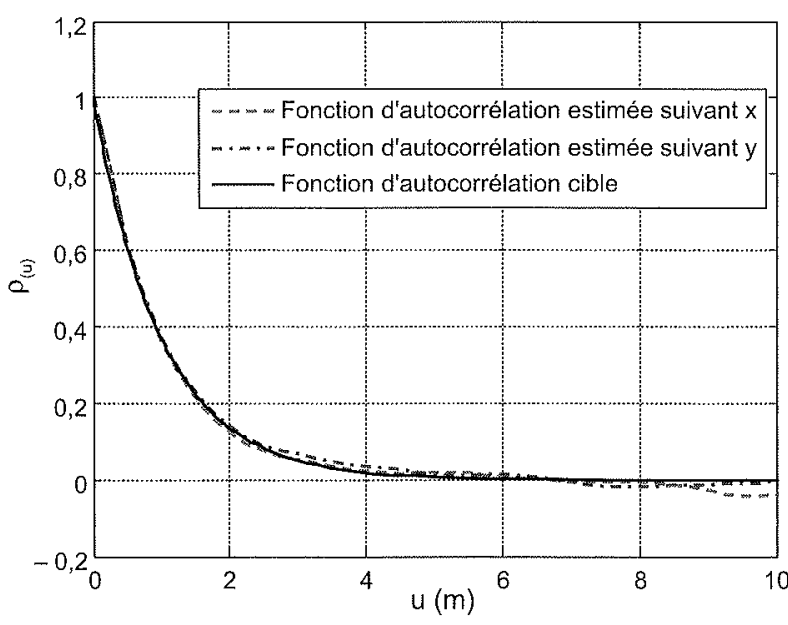

H6. 3 Comparaison entre la fonction d'autocorrélation cible et celle générée du champ de la cohésion suivant $x$ et $y$. Comparison between the target and the generated autocorrelation function of the cohesion random field in the $\mathrm{x}$ and $\mathrm{y}$ directions.

où $\mathrm{j}=0,1, \ldots, \mathrm{M}-1, \mathrm{X}_{\mathrm{k}}$ est la valeur discrétisée du champ à l'intervalle $\mathrm{k} \Delta \mathrm{x}(\mathrm{si} \mathrm{u}=\mathrm{x})$ ou $\mathrm{k} \Delta \mathrm{y}(\mathrm{si} \mathrm{u}=\mathrm{y})$. $\hat{u}_{\mathrm{x}}$ et $\hat{\sigma}_{\mathrm{x}}$ sont respectivement la moyenne et l'écart-type estimés du champ discrétisé. Une bonne concordance entre la fonction d'autocorrélation cible et celle générée est observée pour une réalisation donnée du champ de la cohésion.

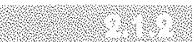

\section{Réalisations du champ de l'angle de frottement}

La figure 4 illustre une réalisation d'un champ isotrope de l'angle de frottement interne suivant une loi de distribution Bêta et dont la distance d'autocorrélation vaut $2 \mathrm{~m}\left(\delta_{x}=\delta_{y}=2 \mathrm{~m}\right)$. La moyenne et le coefficient de variation de l'angle de frottement interne $\varphi$ adoptés dans le calcul sont 30 degrés et $10 \%$ respectivement. Les régions foncées correspondent à des petites valeurs de $\varphi$ et celles plus claires à des valeurs plus grandes. Les valeurs varient entre 18 et 39 degrés. La figure 5 montre la comparaison entre la fonction de probabilité cumulative cible et celle générée, et la figure 6 une autre comparaison entre la fonction d'autocorrelation cible suivant une direction donnée et celle générée et ce, dans les deux directions $\mathrm{x}$ et $\mathrm{y}$, pour une réalisation donnée du champ de l'angle de frottement interne. Comme pour le champ de la cohé-

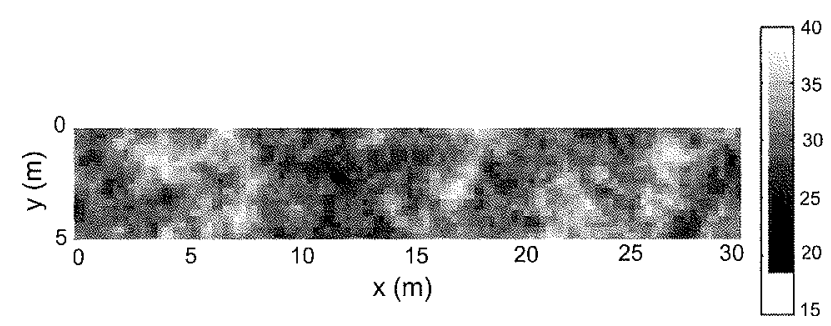

NiG. 4 Réalisation du champ de l'angle de frottement interne (degrés).

Sample of the internal friction angle random field (degrees). 


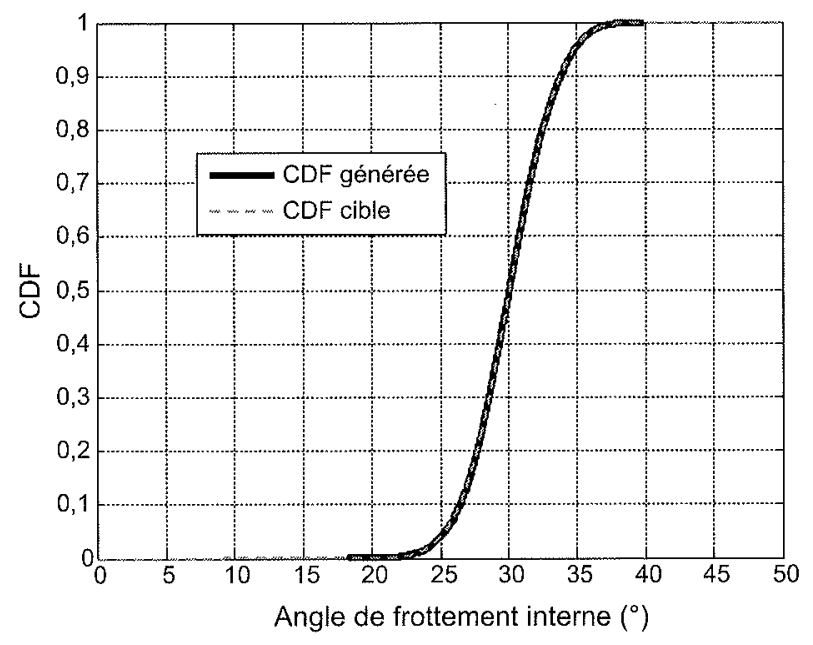

Fic.s Comparaison entre la fonction de répartition (CDF) cible et celle générée du champ de l'angle de frottement interne. Comparison between the target and the generated cumulative distribution function (CDF) of the internal friction angle random field.

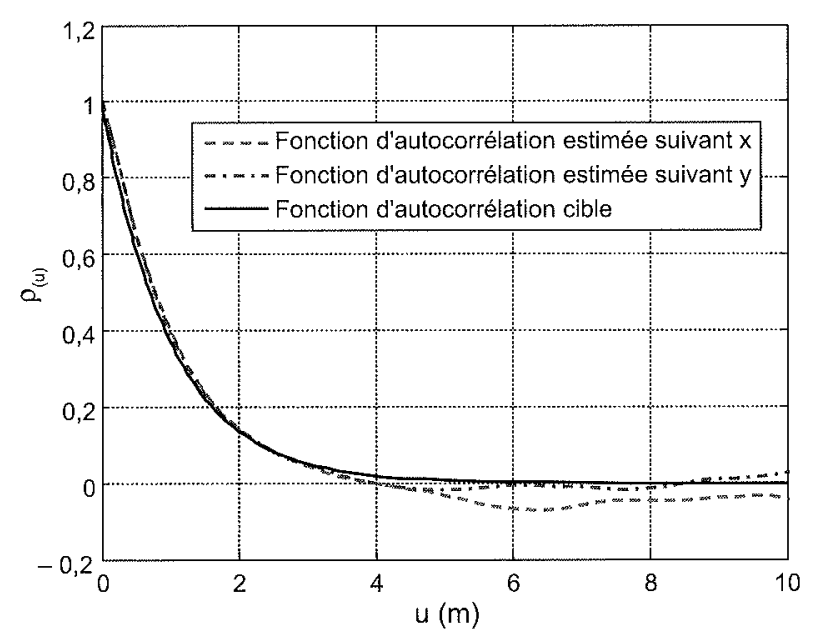

76.6 Comparaison entre la fonction d'autocorrélation cible et celle générée du champ de l'angle de frottement interne suivant $x$ et $y$.

Comparison between the target and the generated autocorrelation function of the internal friction angle random field in the $\mathrm{x}$ and y directions.

sion, on remarque ici une bonne concordance entre les deux fonctions de probabilité cumulative (CDF) et entre les deux fonctions d'autocorrélation (i.e. cible et celle générée) pour une réalisation donnée du champ.

\section{merese \\ Réalisations d'un champ anisotrope}

La figure 7 présente une réalisation d'un champ de cohésion anisotrope (i.e. $\delta_{x}=10 \mathrm{~m} ; \delta_{y}=1 \mathrm{~m}$ ). La moyenne et le coefficient de variation de la cohésion adoptés dans le calcul valent $20 \mathrm{kPa}$ et $20 \%$. Ce type de champ anisotrope est utile pour la modélisation des sols stratifiés fréquemment rencontrés dans la pratique.

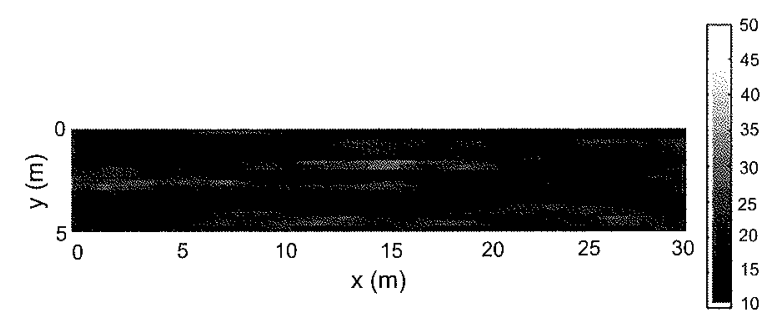

HG T Réalisation d'un champ de cohésion anisotrope $\left(\delta_{\mathrm{y}}=1 \mathrm{~m} ; \delta_{\mathrm{x}}=10 \mathrm{~m}\right)$.

Sample of an anisotropic cohesion random field $\left(\delta_{y}=1 \mathrm{~m} ; \delta_{\mathrm{x}}=10 \mathrm{~m}\right)$.

3

\section{Modèle probabiliste de calcul de la réponse du système}

La réponse du système envisagée dans cet article concerne la charge ultime de la fondation. Le modèle déterministe utilisé est basé sur des simulations numériques sous FLAC ${ }^{3 \mathrm{D}}$. Un pilotage en déplacements est adopté pour le calcul de la charge ultime de la fondation. Un domaine de sol de longueur $15 \mathrm{~B}=30 \mathrm{~m}$ et de hauteur 2,5 B $=5 \mathrm{~m}$ est considéré, $\mathrm{B}$ étant la largeur de la fondation (Fig. 8). Les limites verticales et horizontales du domaine sont suffisamment loin de la fondation pour ne pas perturber le champ de vitesses dans le sol pour toutes les configurations de sol étudiées ultérieurement. Le choix des dimensions des mailles du domaine d'éléments finis a été effectué de façon à conserver la distribution et la structure d'autocorrélation initiale du champ. Pour cela, les dimensions des mailles dépendent des distances d'autocorrélation des propriétés du sol. Der Kiureghian et Ke (1988) ont suggéré que la largeur maximale d'une maille doit être égale à 0,5 fois la distance d'autocorrélation. Puisque la distance d'autocorrélation verticale est de l'ordre de 1 à $4 \mathrm{~m}$, la limite maximale de la dimension verticale de la maille est de l'ordre de $50 \mathrm{~cm}$. En supposant que les distances d'autocorrélation horizontales sont généralement supérieures à $2 \mathrm{~m}$, une limite maximale de $1 \mathrm{~m}$ est imposée pour la dimension horizontale de la maille. Suite aux suggestions proposées ci-dessus, pour choisir les dimensions des mailles dans FLAC ${ }^{3 D}$, une étude a été réalisée pour une fondation superficielle filante de largeur $\mathrm{B}=2 \mathrm{~m}$ reposant sur un sol homogène de caractéristiques, $\mathrm{c}=20 \mathrm{kPa}, \varphi=30$ degrés, $\psi=20$ degrés $;=240 \mathrm{MPa} ; \nu=0,3$ et $\gamma=18 \mathrm{kN} / \mathrm{m}^{3}$. Un maillage non uniforme composé de 1620 zones a été construit. Dans ce maillage, le sol est divisé horizontalement en 30 zones au-dessous de la fondation et 30 zones de part et d'autre de la fondation. Ces zones augmentent progressivement en s'éloignant des extrémités de la fondation jusqu'à ce qu'elles deviennent constantes et égales à $85 \mathrm{~cm}$ dans les régions loin de la fondation pour rester inférieures à la limite maximale (i.e. $1 \mathrm{~m}$ ) proposée par Der Kiureghian et Ke (1988). Verticalement, le domaine est divisé en 18 zones raffinées à proximité de la fondation. Ces mailles augmentent en s'éloignant de la fondation jusqu'à ce que leur largeur verticale devienne constante et égale à $50 \mathrm{~cm}$ dans les zones profondes loin de la fondation pour ne pas dépasser la limite maximale (i.e. $50 \mathrm{~cm}$ verticalement) proposée par Der Kiureghian et Ke (1988). Les conditions aux limites du maillage consistent à bloquer : 
- les déplacements dans la direction perpendiculaire au plan de la figure 8 car le problème traité est bidimensionnel ;

- bloquer les déplacements dans la direction horizontale pour les deux plans verticaux les plus éloignés de la fondation (i.e. limites verticales du maillage) ;

- bloquer tout mouvement dans toutes les directions pour la frontière inférieure du domaine.

Un modèle élastique parfaitement plastique basé sur le critère de rupture de Mohr-Coulomb est utilisé pour représenter le sol. Une fondation de largeur $2 \mathrm{~m}$ et de hauteur $0,5 \mathrm{~m}$ est simulée par un matériau élastique linéaire et non pesant. Pour ce qui concerne le module d'Young de la fondation, une valeur cent fois plus élevée que celle du sol a été adoptée afin de modéliser une fondation rigide. L'interaction sol/fondation est représentée par des éléments d'interface suivant la loi de Coulomb. Les caractéristiques mécaniques de l'interface sont considérées égales à celles du sol, afin de modéliser un contact parfaitement rugueux.

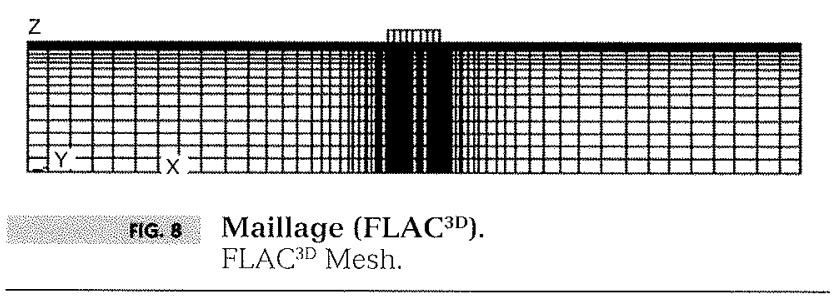

Pour les valeurs moyennes des propriétés du sol et de l'interface, la valeur de la charge ultime calculée en utilisant une vitesse de $5.10^{-6} \mathrm{~m} /$ timestep, est de $2386 \mathrm{kN} / \mathrm{m}$. Le temps de calcul correspondant est de 10 minutes environ sur un ordinateur Pentium IV Centrino 2,0 GHz.

Il est à noter que le maillage stochastique est soumis à plusieurs conditions liées à la fréquence de coupure, aux dimensions du maillage fréquentiel et spatial, etc. Par ailleurs, le domaine et le maillage du modèle mécanique sous FLAC ${ }^{3 \mathrm{~b}}$ doivent être choisis de manière à respecter certaines conditions (mailles fines dans les zones proches des extrémités de la fondation, etc.). Par conséquent, une différence existe entre le maillage stochastique et le maillage de FLAC ${ }^{3 D}$. Un mode de transfert des données stochastiques vers le maillage de FLAC ${ }^{3 D}$ est donc nécessaire. Deux méthodes de transfert existent :

- la méthode du milieu utilisée par Shinozuka et Dasgupta (1987), Yamazaki et Shinozuka (1988), Der Kiureghian et Ke (1988) et Deodatis (1989). Dans cette méthode, le champ aléatoire est représenté par sa valeur au centroïde des mailles du maillage aux éléments finis. On cherche la position du centroïde de chaque maille dans le maillage stochastique et on affecte à la maille d'élément fini la valeur du champ correspondant à la position de son centroïde dans le maillage stochastique;

- la méthode du moyennage local (ou moyennage spatial) proposée par Vanmarcke (1977) et Vanmarcke et Grigoriu (1983). Elle consiste à affecter à chaque maille du domaine aux éléments finis une valeur déterminée par moyennage des valeurs du champ du maillage stochastique qui se situent à l'intérieur de la maille d'élément fini.
Popescu (1995) a effectué une étude comparative entre ces deux méthodes et a conclu que la méthode du milieu est plus adaptée pour des champs non gaussiens puisqu'elle conserve la distribution de probabilité du champ initial. Der Kiureghian et Ke (1988) ont fait remarqué que la méthode du milieu sur-représente la variabilité du champ alors que la méthode du moyennage local tend à sous-représenter la vraie variabilité du champ. Ils ont aussi démontré que la méthode de moyennage spatial est plus adaptée aux champs gaussiens. Dans cette étude, la méthode du milieu a été utilisée puisque les champs aléatoires représentant les propriétés du sol sont considérés comme des champs non gaussiens. Les champs sont générés dans l'environnement de Matlab 7.0. Le transfert entre Matlab 7.0 et FLAC ${ }^{3 \mathrm{D}}$ est effectué par l'intermédiaire de fichiers textes et en utilisant le langage FISH propre à FLAC ${ }^{3 D}$.

\section{3.}

\section{Simulations de Monte-Carlo}

Pour chaque jeu de données des paramètres statistiques des champs aléatoires représentant les propriétés du sol, plusieurs réalisations de champs sont générées dans Matlab 7.0 en utilisant la méthode de représentation spectrale et les simulations de MonteCarlo. Le calcul de la charge ultime est effectué dans FLAC $^{3 D}$ pour chaque réalisation des champs représentant les propriétés du sol. La moyenne et l'écart-type non biaisé de la réponse $R$ sont calculés comme suit:

$$
\begin{gathered}
\mu_{R}=\frac{1}{n_{\text {sim }}} \sum_{i=1}^{n_{\text {sim }}} R_{i} \\
\sigma_{R}=\sqrt{\frac{1}{n_{\text {sim }}-1} \sum_{i=1}^{n_{\text {sim }}}\left(R_{i}-\mu_{R}\right)^{2}}
\end{gathered}
$$

où $\mathrm{n}_{\text {sim }}$ est le nombre de simulations de Monte-Carlo et $\mathrm{R}_{\mathrm{i}}$ est la réponse de la ième simulation de Monte Carlo avec $\left(\mathrm{i}=1, \ldots, \mathrm{n}_{\mathrm{sim}}\right)$. Le nombre de réalisations (correspondant au nombre de calculs déterministes sous $F_{L A C}{ }^{3 D}$ ) nécessaire est celui pour lecuel les valeurs de la réponse moyennée sur l'ensemble des réalisations et de son coefficient de variation convergent.

\section{4 \\ Résultats numériques}

Les résultats numériques sont obtenus pour un sol dont la cohésion c suit une loi lognormale et l'angle de frottement interne $\varphi$ une loi Bêta (Fenton et Griffiths, 2003). Les valeurs choisies pour les moments statistiques des paramètres de résistance au cisaillement du sol et de leur coefficient de corrélation $\rho_{c}$ valent: $\mu_{c}=20 \mathrm{kPa}, \mu_{\varphi}=30$ degrés, $10 \leq \mathrm{COV}_{c}^{\mathrm{c}, \varphi} \leq 40 \%$, $5 \% \leq \mathrm{COV}_{\varphi} \leq 15 \%$ et $\rho_{c, \varphi}=0$. L'angle de dilatance est considéré égal à $2 \varphi / 3$. Les distances de corrélations des propriétés de cisaillement sont considérées égales à $\delta_{x}=\delta_{y}=2 \mathrm{~m}$. La convergence est atteinte à partir d'un nombre de simulations proche de 100 pour lequel l'estimateur de la moyenne de la réponse du système et son coefficient de variation deviennent constants (Figs. 9 et 10). Pour ces 100 simulations, l'intervalle de confiance sur la valeur moyenne de la charge ultime ( \pm un écart-type) est égal à l'écart-type de la charge ultime des 100 simu- 
lations multiplié par $1 / \sqrt{n_{\text {sim }}}=0,1$, ce qui est équivalent

à $10 \%$ de $\sigma_{\mathrm{R}}$. De même, l'intervalle de confiance sur la variance de la charge ultime est égal à la variance de la charge ultime des 100 simulations multipliée par

$\sqrt{\left(2 /\left(n_{\text {sim }}-1\right)\right)}=0,142$, ce qui est équivalent à $14 \%$ de $\sigma_{\mathrm{R}}^{2}$.

Ceci signifie que les valeurs de la moyenne et de l'écart-type des 100 simulations de la charge ultime sont estimées avec un intervalle d'erreur de $\pm 10 \%$ et $\pm 14 \%$ respectivement par rapport aux valeurs réelles. Notons que le temps de calcul nécessaire pour la génération des 100 simulations et le calcul mécanique correspondant est voisin de 20 heures. Ce temps est important mais a déjà fait l'objet d'une optimisation lors du choix du maillage, de la vitesse de pilotage de déplacement et du nombre des simulations de Monte-Carlo, afin d'avoir le meilleur compromis entre la précision et le temps de calcul.

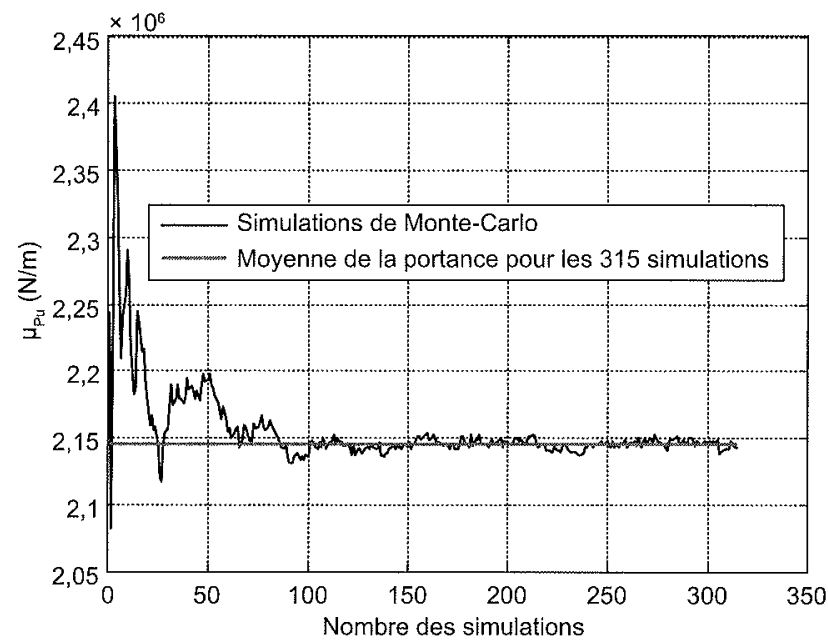

F6.9. Moyenne de la charge ultime en fonction du nombre des simulations $\left(\operatorname{COV}_{\mathrm{c}}=\mathbf{4 0} \%\right.$, $\operatorname{COV}_{\varphi p}=10 \%$ et $\delta_{y}=\delta_{x}=2 \mathrm{~m}$ ).

Mean of the ultimate footing load versus the sample size simulations $(\mathrm{COV}=40 \%$, $\operatorname{COV}_{4 q}=10 \%$ and $\delta_{y}=\delta_{x}=2 \mathrm{~m}$ ).

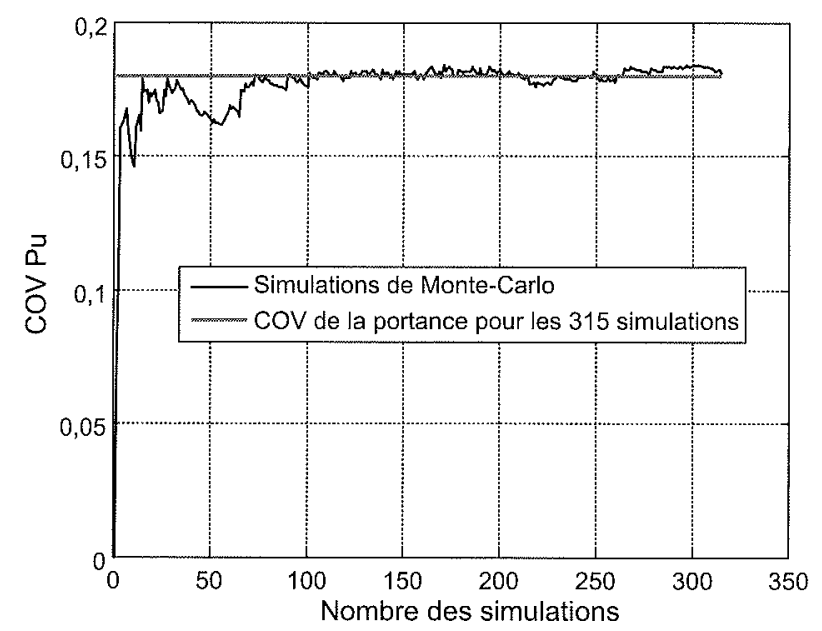

H6. 10 Coefficient de variation de la charge ultime en fonction du nombre des simulations. Coefficient of variation of the ultimate footing load versus the sample size.
Les figures 11 et 12 montrent les courbes effortdéplacement et effort-rotation de toutes les simulations de Monte-Carlo ainsi que les courbes moyennes respectives. Ces dernières sont obtenues en moyennant pour chaque cycle de calcul toutes les valeurs des charges, des déplacements et des rotations obtenues à partir de toutes les simulations.

La valeur de la moyenne de la charge ultime (Fig. 11) est de $2143,5 \mathrm{kPa}$. Cette valeur est inférieure à la valeur déterministe (i.e. $2386 \mathrm{kN} / \mathrm{m}$ ) calculée pour un sol homogène dont les caractéristiques de cisaillement sont constantes et égales aux valeurs moyennes (i.e. $\mathrm{c}=20 \mathrm{kPa}, \varphi=30$ degrés, $\psi=20$ degrés).

Sur la figure 12, on remarque que la courbe moyenne de toutes les simulations correspond à une rotation nulle de la fondation : on retrouve le cas d'un sol homogène pour lequel la fondation reste horizontale et ne subit aucune rotation.

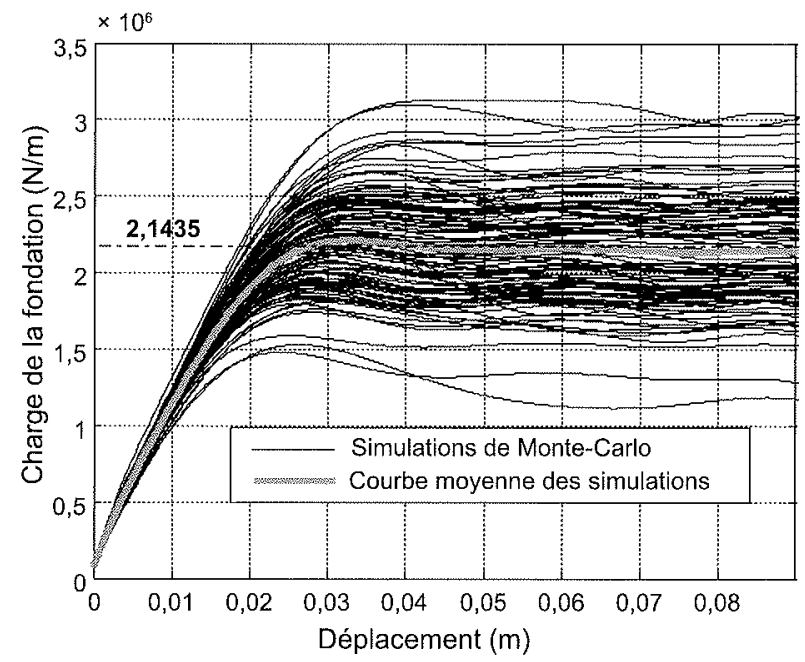

F1. 11 Courbes effort-déplacement des simulations de Monte-Carlo.

Load-displacement curves from Monte-Carlo simulations.

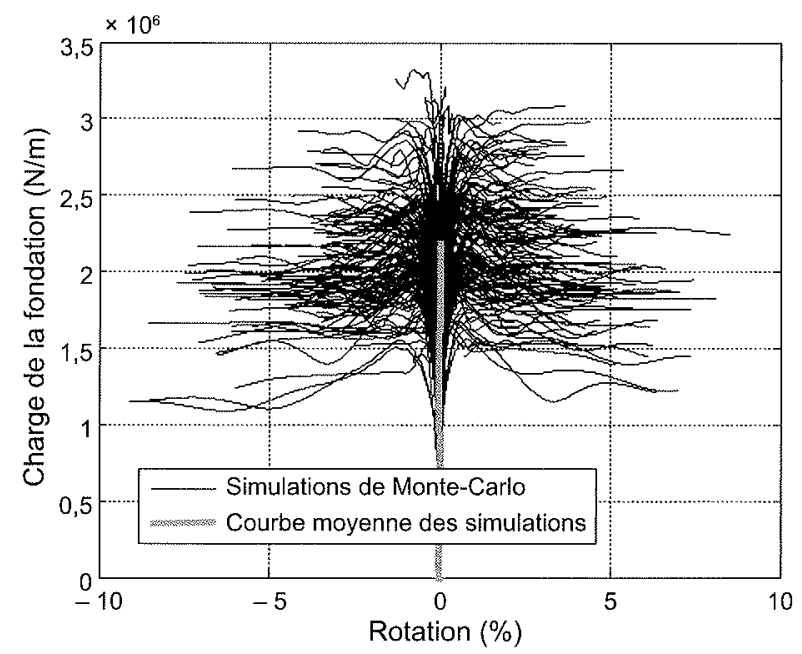

116. 12. Courbes effort-rotation des simulations de Monte-Carlo.

Load-slope curves from Monte-Carlo simulations. 
La figure 13 présente le maillage déformé correspondant à une simulation donnée (i.e. une réalisation des champs de la cohésion et de l'angle de frottement interne). On remarque que la variabilité spatiale correspondant à une réalisation donnée du champ aléatoire crée une asymétrie dans le champ de vitesses et de déformations et aboutit à une rotation de la fondation. Cependant, la moyenne des rotations de l'ensemble des réalisations est nulle comme signalé dans le paragraphe précédent.

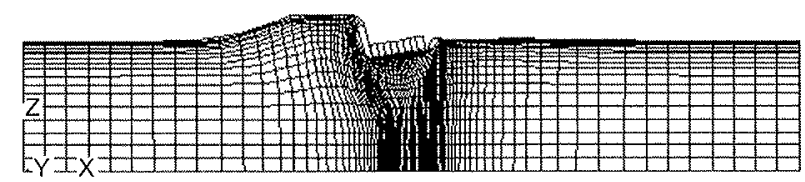

16. 13 Maillage déformé d'une réalisation.

Deformed mesh obtained from a sample of the random field.

A partir des simulations de Monte-Carlo, on peut ajuster l'histogramme des valeurs de la charge ultime de toutes les simulations selon une loi de distribution de probabilité connue. Les lois utilisées sont les lois normale, lognormale et gamma (Fig. 14). La loi lognormale ajuste au mieux l'histogramme de la charge ultime. Il est à noter que, dans le cadre de ce travail où le nombre de simulations de Monte-Carlo est faible, seuls les moments statistiques (moyenne et écart-type) issus de la distribution de la réponse sont exploitables; les queues des distributions de probabilité ajustées ne sont pas rigoureuses. Un nombre supérieur de simulations de Monte-Carlo est nécessaire pour minimiser l'erreur au niveau de ces queues de distribution et pouvoir les exploiter dans le calcul de la probabilité de ruine.

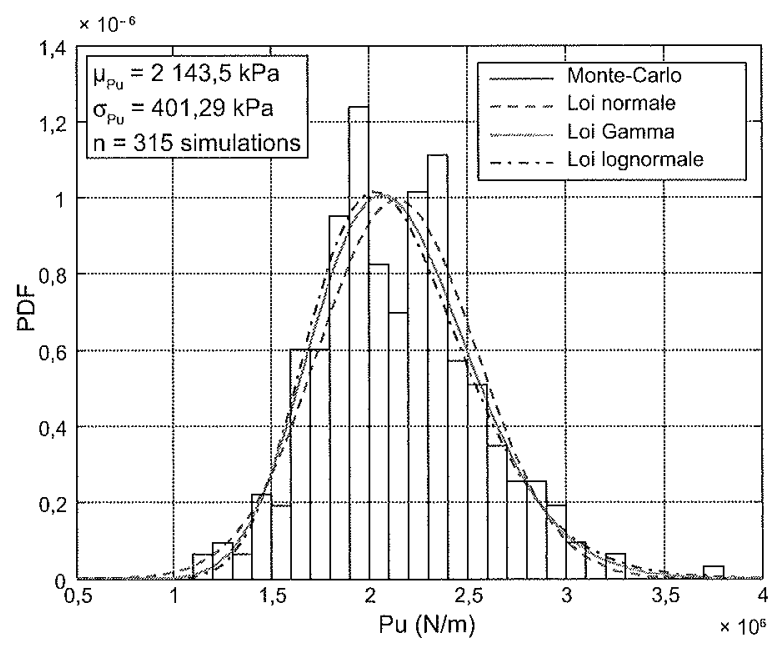

HG. 14 Approximation de la densité de probabilité (PDF) des charges ultimes obtenues à partir des simulations de Monte-Carlo. Probability density (PDF) fitting of the ultimate footing loads obtained by the Monte-Carlo simulations

Les figures 15 et 16 montrent la moyenne et l'écarttype de la charge ultime de la fondation calculés à partir des simulations de Monte-Carlo dans le cas d'un sol isotrope pour lequel la distance d'autocorrélation est supposée la même dans les directions horizontale et verticale.
La variation de la moyenne de la charge ultime de la fondation écrite sous forme adimensionnelle $\mu_{\mathrm{P}} / \gamma \mathrm{B}^{2}$ en fonction de la distance d'autocorrélation pour différentes valeurs des coefficients de variation de la cohésion et de l'angle de frottement interne (Fig. 15) montre que la moyenne de la charge ultime diminue puis augmente avec l'augmentation de la distance d'autocorrélation. Un cas critique existe quand la distance d'autocorrélation devient égale à la largeur de la fondation (i.e. $\delta / \mathrm{B}=1$ ). Ce cas correspond à la plus petite moyenne de la charge ultime. Des résultats similaires ont été observés par Griffiths et Fenton dans plusieurs problèmes de géotechnique (portance des fondations, stabilité des talus). Ceci peut être expliqué comme suit : le mécanisme de ruine cherche à passer par les zones les plus faibles du sol. Cependant, pour une grande variabilité (i.e. des petites distances d'autocorrélation), le mécanisme de ruine rencontre des difficultés pour trouver le chemin le plus faible puisque les zones faibles du sol sont entourées par des zones fortes à cause de la grande variabilité du sol. La ligne de rupture d'un sol présentant une grande variabilité cherche à passer par les zones les plus faibles (i.e. à faibles paramètres de cisaillement). Ceci rend cette ligne de glissement trop tortueuse et, par conséquent, elle est trop ( longue » aboutissant ainsi à une forte charge ultime. Quand la distance d'autocorrélation est égale à la largeur de la fondation, la variabilité du sol est telle que le mécanisme de rupture peut se développer en passant par les zones les plus faibles sans être très étendu en volume. La valeur de la moyenne de la charge ultime est donc plus faible que celle obtenue pour un sol présentant une forte variabilité.

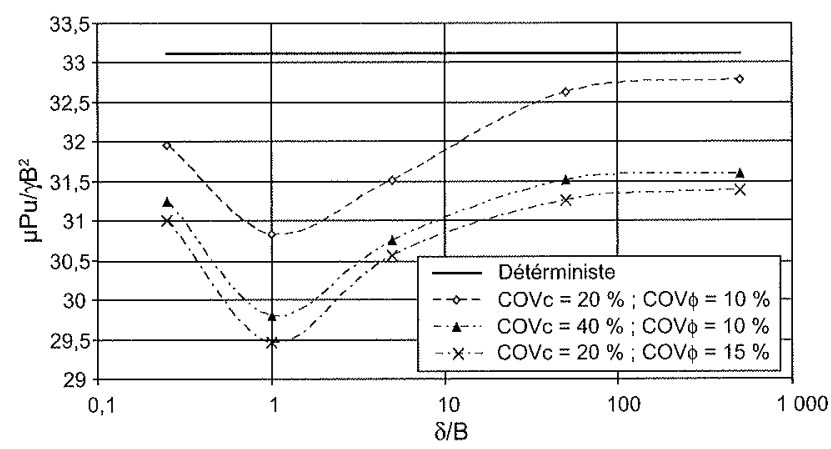

16. 15 Moyenne de la charge ultime en fonction des distances d'autocorrélation.

Mean value of the ultimate footing load versus the autocorrelation distances.

La valeur de la moyenne de la charge ultime augmente avec la diminution des coefficients de variation des paramètres de cisaillement du sol. Elle tend vers la valeur déterministe pour des petits coefficients de variation et de grandes distances d'autocorrélation (i.e. cas d'un sol homogène dont les paramètres de cisaillement sont égaux à leur valeur moyenne).

Concernant l'écart type de la charge ultime (Fig. 16), il augmente avec l'accroissement des distances d'autocorrélation et des coefficients de variation des paramètres de cisaillement du sol.

A partir des figures 15 et 16 , en comparant l'effet d'une augmentation de $5 \%$ du coefficient de variation de l'angle de frottement interne à celui de l'augmentation de $20 \%$ du coefficient de variation de la cohésion, on remarque que la moyenne de la charge ultime et son écart-type sont plus sensibles aux variations de l'angle de frottement interne qu'à celles de la cohésion. 


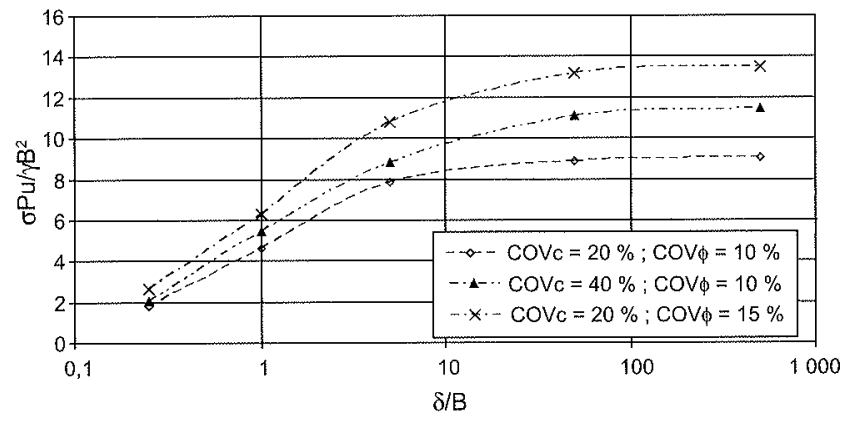

H6. 16 Écart-type de la charge ultime en fonction des distances d'autocorrelation. Standard deviation of the ultimate footing load versus the autocorrelation distances.

\section{Conclusion}

Une analyse probabiliste à l'ELU d'une fondation superficielle filante a été présentée. Posée sur un sol présentant une variabilité spatiale, la fondation a été soumise à un chargement vertical centré. Les propriétés du sol sont modélisées par des champs aléatoires. La méthode de discrétisation de champ utilisée est la méthode de représentation spectrale à l'aide de trans-

formations de Fourrier. Il a été montré que la variabilité spatiale du sol crée une asymétrie dans le champ de vitesses et de déformations et aboutit à une rotation de la fondation pour une réalisation donnée du champ aléatoire. Cependant, la rotation moyenne de la fondation, issue de l'ensemble des simulations de Monte-Carlo, est nulle. La loi lognormale est celle qui ajuste au mieux l'histogramme de la charge ultime de la fondation, obtenu à partir des simulations de MonteCarlo. La valeur de la moyenne de la charge ultime de toutes les simulations est inférieure à la valeur déterministe calculée pour un sol homogène dont les caractéristiques de cisaillement sont constantes et égales aux valeurs moyennes. La moyenne de la charge ultime diminue, puis augmente avec l'accroissement des distances d'autocorrélation pour un sol isotrope. Un minimum existe pour une distance d'autocorrélation égale à la largeur de la fondation. La charge ultime est plus sensible aux variations de l'angle de frottement interne qu'à celles de la cohésion. Enfin, il est à noter que, dans cette étude où le nombre de simulations de Monte-Carlo est faible, seuls les moments statistiques (moyenne et écart-type) issus de la distribution de la réponse sont exploitables; les queues des distributions de probabilité ajustées ne sont pas rigoureuses. Un nombre supérieur de simulations de Monte-Carlo est nécessaire pour minimiser l'erreur au niveau de ces queues de distribution et pouvoir les exploiter dans le calcul de la probabilité de ruine de la fondation vis-àvis d'une rupture par poinçonnement du sol. 
Assimaki D., Pecker A., Popescu R., Prevost J. - Effects of spatial variability of soil properties on surface ground motion. Journal of Earthquake engineering, 7 (1), 2002, p. 1-44.

Deodatis G. - Stochastic FEM sensitivity analysis of nonlinear dynamic problems. Probabilistic Engineering Mechanics, 4 (3), 1989, p. 135-141

Der Kiureghian A., Ke J.-B. - The stochastic finite element method in structural reliability. Probabilistic Engineering Mechanics, 3 (2), 1988, p. 83-91.

Fenton G.A., Griffiths D.V. - Bearing capacity prediction of spatially random $\mathrm{C}-\varphi$ soils. Can. Geotech. J., 40, 2003, p. 54-65.

Fenton G.A. - Estimation for stochastic soil models. J. of Geotech. \& Geoenv. Engrg, ASCE, 125 (6), 1999, p. 470-485.

Fenton G.A., Griffiths D.V. - Probabilistic foundation settlement on spatially random soil. J. of Geotech. \& Geoenv. Engrg, ASCE, 128 (5), 2002, p. 381-390.

Fenton G.A., Griffiths D.V. - Three-Dimensional probabilistic foundation settlement. J. of Geotech. \& Geoenv. Engrg, ASCE, 131 (2), 2005, p. 232-239.

Griffiths D.V., Fenton G.A. - Bearing capacity of spatially random soil : the undrained clay Prandtl problem revisited. Géotechnique, 51 (4), 2001, p. 351-359.

Griffiths D.V., Fenton G.A., Manoharan N. - Bearing capacity of rough rigid strip footing on cohesive soil : Probabilistic study. J. of Geotech. \& Geoenv. Engrg, ASCE, 128 (9), 2002, p. 743-755.

Griffiths D.V., Fenton G.A. - Seepage beneath water retaining structures founcled on spatially random soil. Géotechnique, 43 (4), 1993, p. 577-587.

Griffiths D.V., Fenton G.A. - Influence of soil strength spatial variability on the stability of an undrained clay slope by finite elements. Geotechnical Special Publications, 101, Slope Stability 2000, The GeoInstitute of the American Society of Civil Engineers.
Kalfa M. - Analyse déterministe et fiabiliste d'une fondation superficielle de forme circulaire vis-à-vis de la rupture par poinçonnement du sol. Master de recherche, université de Nantes, 2006.

Popescu R. - Stochastic variability of soil properties : data analysis, digital simulation, effects on system behaviour. $\mathrm{PhD}$ thesis, Princeton University, Princeton, NJ, 1995.

Popescu R., Deodatis G., Nobahar A. Effect of random heterogeneity of soil properties on bearing capacity. Prob. Engrg. Mech., 20, 2005, p. 324-341.

Popescu R., Deodatis G., Prevost J.H. - Simulation of homogeneous nonGaussian stochastic vector fields. Prob. Engrg. Mech., 13 (1), 1998, p. 1-13.

Shinozuka M., Deodatis G. - Simulation of stochastic processes by spectral representation. Applied Mechanics Reviews, ASME, 44 (4), 1991, p. 191-204.

Shinozuka M., Dasgupta G. - Stochastic fields and their digital simulation. Stochastic Methods in Structural Dynamics, G.I. Schueller and M. Shinozuka, eds, Martinus Nijhoff Publishers, Boston, Mass., 1987, p. 93-133.

Soubra A.-H. - Reliability-based analysis and design of eccentrically loaded footings. International Foundation Congress \& Equipment Expo, IFCEE '09, March 15-19 2009, Florida, USA, 8 p.

Soubra A.-H., Youssef Abdel Massih D. Reliability-based analysis and design of obliquely loaded footings. GeoDenver 2007, New Peaks in Geotechnics, ASCE, GSP n 170, Denver, USA, 1821. February 2007, $10 p$

Soubra A.-H., Youssef Abclel Massih D. Reliability-based analysis and design of foundations resting on a spatially random soil. Geocongress 2008, ASCE, GSP $\mathrm{n}^{\circ} 178$, New Orleans, Louisiana, USA, 9-12 March 2008, p. 82-89

Soubra A.-H., Youssef Abdel Massih D. Kalfa M. - Bearing capacity of foundations resting on a spatially random soil. Geocongress 2008, ASCE, GSP $n^{\circ} 178$, New Orleans, Louisiana, USA, 912 March 2008, p. 66-73.

Vanmarcke E. - Probabilistic modeling of soil profiles. Journal of Geotechnical Engineering, ASCE, 103, n GT11, 1977 , p. 1227-1246

Vanmarcke E. - Random fields : analysis and synthesis. MIT press Cambridge, 1983, $382 \mathrm{p}$.

Vanmarcke E., Grigoriu M. - Stochastic finite element analysis of simple beams. Journal of Engineering Mechanics, 109 (5), 1983, p. 1203-1214.

Yamazaki F., Shinozuka M. - Digital generation of non-Gaussian stochastic field Journal of Engineering Mechanics, ASCE, 114 (7), 1988, p. 1183-1197.

Youssef Abdel Massih D. - Analyse du comportement des fondations superficielles filantes par des approches fiabilistes. Thèse de doctorat, université de Nantes, 2007, $269 \mathrm{p}$

Youssef Abdel Massih D., Soubra A.-H., Low B.K. - Reliability-based analysis and design of strip foundations against bearing capacity failure. Journal of Geotechnical and Geoenvironmental engineering, ASCE, 134 (7), 2008a, p. 917-928.

Youssef Abdel Massih D., Kalfa M., Soubra A.-H. - Reliability-based analysis of circular footings using response surface methodology. 2nd BGA Int. Conf. on Foundations, Dundee, 24-27 June 2008b, p. 1625-1636.

Youssef Abdel Massih D., Soubra A.-H. Reliability-based analysis of strip footings using response surface methodology. 10th Int. Conf. on Applications of Statistics and Probability in Civil Engineering, ICASP10, July 31-August 12007 , University of Tokyo, Tokyo, Japan, 8 p.

Youssef Abdel Massih D., Soubra A.- H. - Reliability-based analysis of strip footings using response surface methodology. International Journal of Geomechanics, ASCE, 8 (2), 2008 p. 134-143. 